\title{
A importância da avaliação dos serviços de farmácia no âmbito hospitalar
}

\author{
The importance of the evaluation of pharmacy services in the hospital scope \\ La importância de la evaluación de los servicios de farmácia en el ámbito hospitalario
}

Recebido: 31/10/2021 | Revisado: 07/11/2021 | Aceito: 10/11/2021 | Publicado: 15/11/2021

\author{
Nathalia Zampronio \\ ORCID: https://orcid.org/0000-0001-6254-5768 \\ Faculdade Integrada Carajás, Brasil \\ E-mail: nathaliazampronio11@gmail.com \\ Leticia Piva \\ ORCID: https://orcid.org/0000-0003-0813-3660 \\ Faculdade Integrada Carajás, Brasil \\ E-mail: leticiapiva27@ hotmail.com
}

\begin{abstract}
Resumo
Os estudos voltados à área de Farmácia Hospitalar, principalmente os direcionados à avaliação de seus serviços, são tidos como temas pouco explorados no meio científico. O objetivo do presente estudo foi analisar a importância da avaliação da qualidade dos serviços de Farmácia Hospitalar por meio de uma pesquisa de finalidade básica estratégica, com objetivo descritivo e exploratório, de abordagem qualitativa, tendo como instrumento de pesquisa a revisão narrativa de literatura, delimitada por pesquisas científicas de autores na área de farmácia hospitalar. Foram analisados trabalhos em um recorte temporal entre 2007 a 2020, em que foi possível identificar nestes estudos bibliográficos, as avaliações de como este processo permite a identificação de erros, o apontamento de indicadores, e consequentemente como permite a melhoria dos mesmos e seu aperfeiçoamento para os padrões mínimos indicados, demonstrando a importância da constante avaliação da prestação destes serviços. Essa análise bibliográfica permitiu estabelecer a eficácia da avaliação das atividades prestadas para a modificação da prestação dos serviços farmacêuticos hospitalares e consequente o aumento da qualidade dos serviços de saúde, ampliando a produção científica deste tema na área temática da farmácia hospitalar.

Palavras-chave: Serviço de farmácia hospitalar; Mecanismos de avaliação da assistência à saúde; Hospital; Indicadores de serviço.
\end{abstract}

\begin{abstract}
Studies aimed at the field of Hospital Pharmacy, especially those aimed at evaluating its services, are considered to be themes that are little explored in the scientific community. The aim of this study was to analyze the importance of evaluating the quality of Hospital Pharmacy services through a strategic basic purpose survey, with a descriptive and exploratory objective, with a qualitative approach, having as a research instrument the narrative literature review, delimited by scientific researches by authors in the field of hospital pharmacy. Works were analyzed in a time frame between 2007 and 2020, in which it was possible to identify, in these bibliographical studies, the evaluations of how this process allows the identification of errors, the indication of indicators, and consequently how it allows the improvement of them and their improvement to the minimum standards indicated, demonstrating the importance of constantly evaluating the provision of these services. This bibliographic analysis made it possible to establish the effectiveness of the evaluation of the activities provided to modify the provision of hospital pharmaceutical services and, consequently, increase the quality of health services, expanding the scientific production on this topic in the thematic area of hospital pharmacy.
\end{abstract}

Keywords: Hospital pharmacy service; Health care evaluation mechanisms; Hospital; Service indicators.

\section{Resumen}

Los estudios dirigidos al campo de la Farmacia Hospitalaria, especialmente los dirigidos a evaluar sus servicios, se consideran temas poco explorados en la comunidad científica. El objetivo de este estudio fue analizar la importancia de evaluar la calidad de los servicios de Farmacia Hospitalaria a través de una encuesta de propósito básico estratégico, con un objetivo descriptivo y exploratorio, con un enfoque cualitativo, teniendo como instrumento de investigación la revisión de la literatura narrativa, delimitada por. investigación científica de autores en el campo de la farmacia hospitalaria. Los trabajos fueron analizados en un período de tiempo comprendido entre 2007 y 2020 , en el cual se pudo identificar, en estos estudios bibliográficos, las evaluaciones de cómo este proceso permite la identificación de errores, la indicación de indicadores, y en consecuencia cómo permite la mejora de ellos y su mejora a los estándares mínimos indicados, demostrando la importancia de evaluar constantemente la prestación de estos servicios. Este análisis bibliográfico permitió establecer la efectividad de la evaluación de las actividades realizadas 
para modificar la prestación de los servicios farmacéuticos hospitalarios y, en consecuencia, incrementar la calidad de los servicios de salud, ampliando la producción científica sobre este tema en el área temática de farmacia hospitalaria.

Palabras clave: Servicio de farmacia hospitalaria; Mecanismos de evaluación de la atención de la salud; Hospital; Indicadores de servicio.

\section{Introdução}

Diante de uma perspectiva histórica, a Farmácia Hospitalar é um setor considerado muito recente na realidade dos hospitais brasileiros. Com a chegada dos portugueses, no cenário de Brasil Colônia, inaugurou-se em 1543 o primeiro hospital no país, a Santa Casa de Misericórdia de Santos. Entretanto, as primeiras farmácias hospitalares tiveram sua formação apenas na década de 1950 (Cavallini \& Bisson, 2010).

Considerando a escassez de informações e referencial teórico que auxiliasse os farmacêuticos na prática hospitalar, criou-se em maio de 1995, a Sociedade Brasileira de Farmácia Hospitalar (SBRAFH), uma entidade nacional que estimula a qualificação profissional dos farmacêuticos hospitalares e os representa junto à sociedade e demais profissionais em saúde (Thomaz, 2005).

Em 1997, após três oficinas de trabalho, a SBRAFH lançou a primeira edição dos Padrões Mínimos para Farmácia Hospitalar e Serviços de Saúde, atualmente conta com sua terceira edição atualizada em 2017. Um referencial de informações fornecidas por especialistas na área, que reúnem seus conhecimentos em prol da qualificação permanente dos profissionais farmacêuticos, visando aprimorar os serviços de assistência farmacêutica nos hospitais (SBRAFH, 2017).

O farmacêutico hospitalar exerce importantes funções clínicas, administrativas e econômicas, mantendo ligação com a direção do hospital e com as demais unidades que prestam assistência ao paciente. Visto a extensa demanda de funções atribuídas a esse profissional, é importante determinar padrões mínimos para a execução dessas atividades, a fim de desempenhar o básico para garantir a segurança na assistência farmacêutica prestada.

O sistema de saúde possui um caráter multiforme, do qual os problemas em saúde e as intervenções necessárias para solucioná-las são cercados de dúvidas. Devido à velocidade com que as tecnologias médicas se desenvolvem e as expectativas da sociedade, deve se considerar a importância da busca por informações que revelem a eficácia do sistema de saúde por meio da avaliação (Contandriopoulos, et al, 1997).

A avaliação permite produzir informações, analisar seus efeitos a curto e longo prazo, além de estabelecer a eficácia dos serviços para a modificação do estado de saúde dos usuários do serviço avaliado. Levando em consideração o contexto da pesquisa, o procedimento escolhido, a natureza da intervenção e o propósito desta avaliação (Contandriopoulos et al, 1997).

Portanto, o presente projeto visa analisar a importância da avaliação da qualidade dos serviços de farmácia hospitalar, desenvolvendo argumentos que justifiquem a relevância dessa avaliação para a melhora dos serviços prestados neste setor no âmbito hospitalar.

\section{Metodologia}

A metodologia que foi utilizada para a construção do presente trabalho se baseia na revisão narrativa de literatura, que nada mais é do que a análise da literatura publicada que traçou um quadro teórico e fez a estruturação conceitual que deu sustentação ao desenvolvimento desta pesquisa (Martins, 2018).

Para elaboração desta revisão de literatura, que é uma pesquisa qualitativa e descritiva que delimitou este trabalho para se embasar em trabalhos publicados nos últimos 14 anos. Foram utilizados livros, sítios de farmácia, revistas eletrônicas, artigos científicos, trabalhos acadêmicos e material científico de farmácia, em distintas bases de dados tais como: Scielo, Medline (PubMed), Periódicos Capes e em repositórios científicos. Escolheu-se como critério a inclusão de documentos de cunho científico, no idioma português. Também foram incluídos documentos legislatórios (governamentais) os mesmos, não 
passaram por seleção prévia de datas para busca. Excluíram-se documentos os quais não apresentaram relação com os objetivos da pesquisa, por meio da leitura de título e resumo. Foram utilizadas as seguintes palavras-chave para busca dos arquivos científicos: Farmácia Hospitalar, Análise em Saúde, Importância da Avaliação, Farmacêutico Hospitalar.

Utilizando os critérios acima, foram encontrados 158 artigos, dos quais 27 possuíam alguma relação com o objetivo traçado, destes 12 foram analisados para o desenvolvimento da revisão, considerando que os mesmos abordassem as temáticas da avaliação dos serviços de farmácia hospitalar, as competências deste setor, e a importância dessa avaliação para a melhoria de indicadores em saúde e dos serviços prestados.

\section{Resultados e Discussão}

\section{Das competências da farmácia hospitalar}

Dentre os diversos setores que compõem a estrutura de um hospital está a Farmácia Hospitalar, ambiente este composto por elementos de gestão e relação multiprofissional. A portaria nº4. 283/2010 do Ministério da Saúde define Farmácia Hospitalar como:

A unidade clínico-assistencial, técnica e administrativa, onde se processam as atividades relacionadas à assistência farmacêutica, dirigida exclusivamente por farmacêutico, compondo a estrutura organizacional do hospital e integrada funcionalmente com as demais unidades administrativas e de assistência ao paciente (Brasil, 2010).

Ao avaliar essa definição é mais compreensível isolar alguns dos elementos para a elucidação adequada. O termo clínico-assistencial refere-se ao fato do farmacêutico hospitalar estar envolvido em atividades de foco clinico que visem à melhora da farmacoterapia do paciente (Neuwien, 2014).

A unidade administrativa está relacionada com as atribuições referentes ao controle de estoque e gestão de custos dos medicamentos e outros itens da farmácia hospitalar. Já a integração com os demais setores e equipe multiprofissional, tem por objetivo um atendimento adequado ao paciente (Neuwien, 2014).

Selecionar medicamentos é um procedimento com envolvimento multiprofissional, que visa economia, segurança e eficácia nos atendimentos de fato necessários à população assistida, portanto deve estar alicerçada em parâmetros epidemiológicos, técnicos e econômicos (Brasil, 2002). Considerando que todo ciclo parte de um princípio, a seleção é o processo inicial do ciclo da assistência farmacêutica. Escolher dentre vários medicamentos e selecionar quais farão parte do elenco da unidade hospitalar é uma atividade minuciosa que exige avaliar diversos fatores, compreendendo sua importância para os processos seguintes.

De acordo com a SBRAFH as atribuições essenciais da Farmácia Hospitalar são: gestão, desenvolvimento de infraestrutura, logística farmacêutica e preparo de medicamentos, otimização da terapia medicamentosa, farmacovigilância e segurança do paciente, informações sobre medicamentos e produtos para saúde, ensino, educação permanente e pesquisa (SBRAFH, 2017).

Todas essas atribuições são administradas por meio de uma direção técnica, que é responsabilidade exclusiva do profissional farmacêutico. Não necessariamente executadas por ele, porém a elaboração, execução e acompanhamento destes processos, visando o funcionamento adequado das ações e adequação das inconformidades.

\section{Avaliação dos serviços de farmácia hospitalar}

Nos estudos de Silva et al (2013) foi analisada a qualidade da prestação dos serviços da farmácia hospitalar em 20 hospitais do estado do Rio de Janeiro no ano de 2009, em que destes 20 hospitais, 19 constavam do organograma da estrutura organizacional da instituição. Em 15 deles $(78,9 \%)$ a farmácia encontrava-se representada como órgão, divisão, departamento, 
serviço, setor ou seção responsável pelos medicamentos. Em três, a farmácia estava vinculada à área administrativa ou de suprimentos, enquanto nos demais encontrava-se hierarquicamente ligada à área clínica. Estes hospitais reuniam, à época, 3.698 leitos, cerca de 6,5\% do total dos hospitais do estado do Rio de Janeiro.

Neste mesmo estudo os indicadores relacionados a "gerenciamento" concluíram que 16 dos 20 hospitais cumpriram com menos de $50 \%$ dos indicadores mínimos necessários, com relação ao indicador de "seleção" apenas uma farmácia cumpriu com $75 \%$ dos indicadores mínimos necessários, em oito faltavam medicamentos considerados essenciais para o controle e o tratamento das principais causas de internação no Estado. O indicador "armazenamento" foi o mais bem sucedido sendo todos os indicadores mínimos cumpridos em 16 dos 20 hospitais.

Problemas relacionados às condições de trabalho, como espaço físico insuficiente, falta de procedimentos por escrito e divergências na distribuição de medicamentos no setor, parecem ser as principais causas dos erros de distribuição encontrados neste estudo, acerca dos Hospitais Estaduais do Rio de Janeiro (Silva, 2013).

Desenvolvendo estudos sobre a importância da avaliação se tem os estudos de Contandriopoulos et al (1997) em que segundo ele a avaliação corresponde ao processo de fazer julgamentos de valor para o objeto avaliado, acompanhando as ações técnicas e seus efeitos esperados, apoiando o processo de tomada de decisão e formulação de políticas, operando por meio de um método sistemático e buscando a melhoria contínua na assistência.

O estudo do referido autor foi aplicado nos hospitais públicos sob gestão da Secretaria de Saúde do Distrito Federal, e trouxe as reflexões de que independentemente da variação quanto à complexidade hospitalar, a baixa porcentagem de adequação das áreas verificada nos resultados (34,6\%; 18 de 52 áreas) chama atenção e reflete a possibilidade de que elas não foram concebidas em termos de instalações e localizações para a execução efetiva dos serviços farmacêuticos (Lima, 2018).

Dentre os serviços avaliados, o gerenciamento foi o que apresentou maior proporção de cumprimento dos itens previstos, foi frequente a ausência de planejamento de objetivos e metas ou programação para capacitação de RH, aspecto que não influenciou positivamente e com significância estatística a execução dos serviços avaliados. Foi observada uma quantidade de leitos por farmacêutico menor do que a evidenciada no Projeto de Diagnóstico da Farmácia Hospitalar no Brasil, porém, superior ao preconizado pela SBRAFH (2017); (Lima, 2018).

Conforme a análise dos estudos de Nascimento (2011), que tem por base análise bibliográfica, observou-se uma melhor situação para os setores de farmácia hospitalar no Brasil em regiões onde existe a associação das categorias de variáveis relacionadas ao módulo gerenciamento como: presença de planejamento de objetivos e metas, maior quantidade de farmacêuticos com especialização, com cursos de atualização e que participaram de congressos e outras conferências. Observando-se que mesmo com orçamentos por vezes inadequados esses hospitais disponibilizam serviços com profissionais qualificados.

Ainda conforme Nascimento (2011) em uma avaliação mundial realizada em 2007 sobre a prática em farmácias hospitalares constatou-se que somente 35\% dos países confirmaram ter o farmacêutico presente ou acessível por 24 horas. De acordo com a Portaria n 4.283 de 30 de dezembro de 2010 (Brasil, 2010) a responsabilidade técnica da farmácia hospitalar é atribuição do farmacêutico, e sua presença em todo ciclo de assistência farmacêutica favorece a garantia da segurança dos pacientes (Nascimento, 2011).

Conforme os estudos de Silva et al (2018), foram analisados quatro hospitais localizados na região Centro-Oeste do Brasil, sendo dois de administração pública direta (G1APD) e dois administrados por Organizações Sociais de Saúde (G2OSS), neste estudo observou-se que todos os Serviços de Farmácia Hospitalar (SFH) adotam o modelo de sistema misto ou combinado de dispensação, tanto para medicamentos quanto para materiais médico-hospitalares. Não foi constatado planejamento escrito de objetivos e metas em nenhum dos SFH, bem como a presença do farmacêutico na constituição do Serviço ou Comissão de Controle de Infecções Hospitalares (SCIH/CCIH) e nas Comissões de Suporte Nutricional (CSN). 
Launé (2019) considerando a carência de instrumentos para avaliação da qualidade dos serviços prestados em farmácia hospitalar realizou a criação de um instrumento de autoavaliação destes serviços, como ferramenta que auxilie os hospitais e gestores no desenvolvimento de serviços de qualidade com foco na acreditação hospitalar. $\mathrm{O}$ instrumento foi validado por meio de pré-teste em quatro hospitais no município de Niterói-RJ e o roteiro foi disponibilizado de forma gratuita para a contribuição nos processos de melhoria interna dos serviços prestados.

Costa et al (2016) realizou a avaliação dos serviços de farmácia de 22 hospitais públicos do estado da Bahia, utilizouse a metodologia do projeto Diagnóstico da Farmácia Hospitalar no Brasil, sendo incluso 62 indicadores com 244 perguntas. Os autores obtivem respostas que apontaram baixo desempenho nos processos de assistência farmacêutica e os serviços administrativos sobressaíram os clínicos de maneira geral. Além de, apontar uma carência profissional para a quantidade de serviços e na qualidade de execução dos mesmos.

\section{Importância da avaliação dos SFH e aplicação para melhoria de indicadores}

A avaliação dos serviços farmacêuticos hospitalares é importante devido ao fato de que a partir deles são desenvolvidos novos mecanismos que auxiliam na melhoria da prestação destes, refletindo de modo geral em todo o serviço de saúde fornecido aos pacientes.

Na revisão integrativa de Damasceno et al (2020) citam que avaliar a qualidade e o desempenho de hospitais por meio da análise de indicadores internos ajuda a compreender plenamente as necessidades de melhoria da unidade hospitalar utilizando-se dados. Neste estudo as autoras abordam a aplicabilidade e contribuições dos principais indicadores hospitalares, que destacam como essas avaliações poder ser fundamentais para os custos hospitalares e o aperfeiçoamento das atividades desempenhadas.

Conclui-se desta maneira que cada unidade hospitalar possui suas especificidades, que envolve critérios como localização, competências para gestão, porte, complexidade, dentre outros, contudo, visando uma padronização na qualidade de prestação dos serviços farmacêuticos. O Ministério da Saúde implementou a Portaria no 4283/2010 do Ministério da Saúde, que aprova as "diretrizes e estratégias para organização, fortalecimento e aprimoramento das ações e serviços de farmácia no âmbito dos hospitais", onde após a avaliação da qualidade dos serviços farmacêuticos estudos demonstram que a aplicação de tais diretrizes visa a melhora significativa destes de maneira uniforme, independente do porte e complexidade hospitalar (Santana et al, 2013).

De acordo com os estudos de Santana et al (2013) após a avaliação dos serviços de farmácia hospitalar foram detectadas falhas e então aplicada uma reestruturação (seguindo as diretrizes da Portaria nº 4283/2010 do Ministério da Saúde) onde as etapas de programação e aquisição de suprimentos passaram a ser realizadas de forma centralizada, apresentando um desempenho considerado "bom ou estruturado" com um percentual de adequação de $75 \%$. O processo de seleção de medicamentos e demais tecnologias foi o componente que apresentou o maior percentual de melhoria de acordo com os parâmetros avaliados (aumento de 73,3\% na adequação às diretrizes do Ministério da Saúde). No que concernem às atividades de armazenamento nas unidades avaliadas, foi observado um incremento significativo de uma média de $15,5 \%$ de adequação para $48,3 \%$.

Com a reestruturação das unidades a um modelo de gestão em rede, observou-se uma melhora na adequação de alguns processos logísticos com destaque para as etapas de seleção, programação, aquisição e armazenamento que obtiveram os melhores percentuais de adequação às diretrizes do Ministério da Saúde. Essas mudanças foram atribuídas principalmente pelo processo de centralização da gestão e concentração de atividades como seleção de medicamentos e aquisição de itens a nível central (Santana et al, 2013).

Magarinos-Torres (2007) destaca a falta de artigos brasileiros que desenvolvam trabalhos em torno da Logística, 
atividade esta que interfere de maneira direta nas decisões clínicas, refletindo a escassez da abordagem desses estudos. Além de que, o autor evidencia que na literatura observa-se que os resultados obtidos na avaliação de diversos serviços de farmácia hospitalar estão em baixa adequação das normas legais e dos padrões esperados.

As constantes avaliações para a melhoria da qualidade dos serviços farmacêuticos são tão importantes para o desenvolvimento de novas estratégias, que de acordo com os estudos de Volkart (2016) no município de Salvador/Bahia, foram comparados os erros de dispensação em uma farmácia hospitalar antes e após inclusão de estratégias de prevenção de erros.

Costa, Valli e Alvarenga (2008) indicam que são poucos os estudos que abordam especificamente os erros de dispensação e os que tratam do assunto apresentam uma taxa acima de 10\%. Em 2009, 29\% das prescrições apresentavam erros, enquanto 4,27\% do total de medicamentos dispensados continha algum erro, já em 2012 após a implementação de estratégias de prevenção, os erros em prescrição foram reduzidos para $11,35 \%$, enquanto a taxa de erros de medicamentos dispensados caiu para 2,74\% (Galvão, 2012).

$\mathrm{O}$ aperfeiçoamento de práticas de gestão da qualidade necessita de uma estrutura organizacional que possibilite a execução do serviço e que requerem estratégias delineadas, atividades integradas e revisão constante de melhorias de técnicas e resultados (Cipriano et al, 2009). A falta de planejamento eficaz das ações realizadas, a falta de manuais de normas e procedimentos e a falta de farmacêuticos e demais profissionais em comissões multiprofissionais afetam a gestão. Portanto, é necessário formular ações norteadoras a serem colocadas em prática.

\section{Considerações Finais}

Entende-se que o desenvolvimento de estudos nesta área é de suma necessidade para apontarem nos hospitais brasileiros quais são os principais pontos do serviço farmacêutico hospitalar que necessitam de melhoria, e este apontamento pode ser feito pela avaliação de qualidade, por isso sua extrema importância.

Dos relatos bibliográficos analisados em muitos casos houve clara dificuldade de prestação de serviços relacionados a distribuição, falta de medicamento e planejamento e organização, verificando-se uma precariedade nestes critérios. Contudo, com as devidas avaliações, estes dados gerarão indicadores que nortearão as futuras estratégias que serão aplicadas nestes hospitais.

Deste mesmo modo, os autores demonstraram que a adequação as diretrizes do Ministério da Saúde e resolução dos pontos negativos das farmácias hospitalares corroboraram para a melhoria da qualidade dos serviços prestados, além disso, também pode se constatar que a partir da avaliação destes serviços e indicação de pontos deficitários desta prestação, houve a adequação e diminuição de erros.

Por fim, a crescente demanda por serviços de saúde mais efetivos, assim como a racionalidade no uso de medicamentos, redução de custos, minimização de problemas relacionados a esse insumo e aumento da segurança da farmacoterapia, fundamentam a importância de um processo constante de avaliação e monitoramento da Assistência Farmacêutica Hospitalar.

\section{Referências}

Brasil. Ministério da Saúde. (2002). Assistência Farmacêutica na Atenção Básica: instruções técnicas para sua organização. http://bvsms.saude.gov.br/bvs/publicacoes/cd03_15.pdf.

Brasil. Ministério da Saúde. (2010). Portaria n n $^{\mathbf{2}}$.283, de 30 de dezembro de 2010. Aprova as diretrizes e estratégias para organização, fortalecimento e aprimoramento das ações e serviços de farmácia no âmbito dos hospitais. Diário Oficial da União. https://bvsms.saude.gov.br/bvs/saudelegis/gm/2010/prt4283_30_12_2010.html.

Cavallini, M. E., \& Bisson, M. P. (2002). Farmácia hospitalar: um enfoque em sistemas de saúde. Manole. 
Cipriano, S. L., Pinto, V. B., \& Chaves, C. E. (2009). Gestão estratégica em farmácia hospitalar: aplicação prática de um modelo de gestão para qualidade. In Gestão estratégica em farmácia hospitalar: aplicação prática de um modelo de gestão para qualidade (pp. 158-158).

Contandriopoulos, A. P., Champagne, F., Denis, J. L., \& Pineault, R. (1997). A avaliação na área da saúde: conceitos e métodos. Avaliação em saúde: dos modelos conceituais à prática na análise da implantação de programas. https://doi.org/10.7476/9788575414033.

Costa, L. A., Santana, G. S., Pinto, C. R., \& Sampaio, B. C. (2016). Diagnóstico dos serviços de farmácia hospitalar da Secretaria da Saúde do Estado da Bahia, Nordeste do Brasil. Jornal de Assistência Farmacêutica e Farmacoeconomia, 1(2), 24-32. doi:10.22563/2525-7323.

Costa, L. A., Valli, C., \& Alvarenga, A. P. (2008). Erros de dispensação de medicamentos em um hospital público pediátrico. Revista Latino-Americana de Enfermagem, 16, 812-817. https://doi.org/10.1590/S0104-11692008000500003

Damasceno, V. A., \& Alves, K. K. A. F. (2020). Aplicação e contribuições dos indicadores hospitalares: uma revisão integrativa. Research, Society and Development , 9 (8). http://dx.doi.org/10.33448/rsd-v9i8.6637.

Galvão, A. A., de Oliveira, A. M., de Carvalho, F. B., \& Araújo, R. P. C. (2012). Identificação e distribuição dos erros de dispensação em uma farmácia hospitalar: um estudo comparativo no município de Salvador Bahia. Revista de Ciências Médicas e Biológicas, 11(2), 201-206.

Launé, L. C. D. S. (2019). Proposta de roteiro de auto-avaliação dos serviços de farmácia hospitalar: um instrumento a luz da qualidade e acreditação.

Lima, R. F. (2018). Avaliação dos serviços farmacêuticos em farmácias hospitalares de hospitais públicos do Distrito Federal-Brasil. https://repositorio.unb.br/bitstream/10482/32156/1/2018_RodrigoFonsecaLima.pdf.

Magarinos-Torres, R., Osorio-de-Castro, C. G. S., \& Pepe, V. L. E. (2007). Atividades da farmácia hospitalar brasileira para com pacientes hospitalizados: uma revisão da literatura. Ciência \& Saúde Coletiva, 12, 973-984.

Martins, M. D. F. M. (2018). Estudos de revisão de literatura. https://www.arca.fiocruz.br/bitstream/icict/29213/2/Estudos_revisao.pdf>.

Nascimento, A. (2011). Avaliação de Farmácias Hospitalares Brasileiras Utilizando Análise de Correspondência Múltipla (Dissertação de mestrado. Rio de Janeiro: UFRJ). http://www.peb.ufrj.br/teses/Tese0134_2011_05_03.pdf.

Neuwien, A. L. F. (2014). Estrutura Organizacional da Farmácia Hospitalar. In: Neuwien, A. L. F. Gerenciamento de Farmácia Hospitalar. UNIASSELVI. 67.

Santana, R. S., Santos, A. D. S., Menezes, M. S., De Jesus, E. M. S., \& Da Silva, W. B. (2013). Assistência farmacêutica de uma rede de hospitais públicos: proposta de utilização das diretrizes ministeriais para avaliação do serviço. Revista Brasileira de Farmácia Hospitalar e Serviços de Saúde, 4(1). http://www.v1.sbrafh.org.br/public/artigos/2013040105BR.pdf.

SBRAFH. (2017). Padrões Mínimos para Farmácia Hospitalar. (3 ed.) Sociedade Brasileira de Farmácia Hospitalar.

Silva, M. J. S. D., Magarinos-Torres, R., Oliveira, M. A., \& Osorio-de-Castro, C. G. S. (2013). Avaliação dos serviços de farmácia dos hospitais estaduais do Rio de Janeiro, Brasil. Ciência \& saúde coletiva, 18, 3605-3620. https://www.scielo.br/j/csc/a/gTpkxfCn3hBXBYLCXSTmgNH/?lang=pt\&format=pdf.

Thomaz, S. (2005). SBRAFH: Dez anos de História. http://www.sbrafh.org.br/documentos/historico.pdf.

Volkart, G. F. (2016). Avaliação de serviço de saúde: estudo exploratório de erros de dispensação em farmácia hospitalar no Rio Grande do Sul. http://hdl.handle.net/10183/183732 